\title{
SnpReady for Rice (SR4R) Database
}

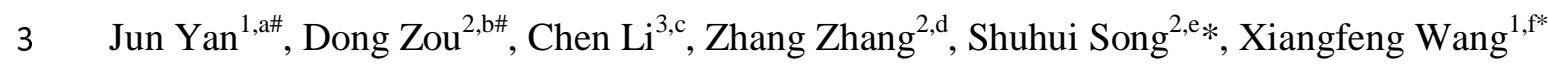

4

$5{ }^{1}$ Department of Crop Genomics and Bioinformatics, College of Agronomy and

6 Biotechnology, China Agricultural University, Beijing 100094, China

$7 \quad{ }^{2}$ National Genomics Data Center \& BIG Data Center \& CAS Key Laboratory of Genome

8 Sciences and Information, Beijing Institute of Genomics, Chinese Academy of Sciences,

9 Bejing 100101, China

$10{ }^{3}$ Rice Research Institute, Guangdong Academy of Agricultural Sciences, Guangzhou 510640,

11 China

$13 \quad$ Equal contribution

$14 *$ Correspondence should be addressed to xwang@ cau.edu.cn (Wang XF) and

15 songshh@big.ac.cn (Song SH);

16 Emails of authors:

17 hh405262448@126.com (Yan J);

$18 \quad$ zoud@big.ac.cn (Zou D);

19 lic11111@sina.com (Li C);

20 zhangzhang@big.ac.cn (Zhang Z)

22 a ORCID: 0000-0002-3806-6457

23 b ORCID: 0000-0002-7169-4965

24 c ORCID: 0000-0001-6702-6860

25 d ORCID: 0000-0001-6603-5060 
26 e ORCID: 0000-0003-2409-8770

27 f ORCID: 0000-0002-6406-5597

28

29 Number of words: 5421

30 Number of figures: 7

31 Number of tables: 0

32 Number of supplementary figures: 4

33 Number of supplementary tables: 3

34

35 Running title: Jun Yan et al. / SNP Ready for Rice database

36

37

38

39 


\section{Abstract}

41 The information commons for rice (IC4R) database is a collection of $\sim 18$ million SNPs 42 (single nucleotide polymorphisms) identified by the resequencing of 5,152 rice accessions. 43 Although IC4R offers ultra-high density rice variation map, these raw SNPs are not readily 44 usable for the public. To satisfy different research utilizations of SNPs for population 45 genetics, evolutionary analysis, association studies and genomic breeding in rice, the raw 46 genotypic data of the 18 million SNPs were processed by unified bioinformatics pipelines. 47 The outcomes were used to develop a daughter database of IC4R - SnpReady for Rice 48 (SR4R). The SR4R presents four reference SNP panels, including 2,097,405 hapmapSNPs 49 after data filtration and genotype imputation, 156,502 tagSNPs selected from linkage 50 disequilibrium (LD)-based redundancy removal, 1,180 fixedSNPs selected from genes 51 exhibiting selective sweep signatures, and 38 barcodeSNPs selected from DNA fingerprinting 52 simulation. SR4R thus offers a highly efficient rice variation map that combines reduced SNP 53 redundancy with extensive data describing the genetic diversity of rice populations. In 54 addition, SR4R provides rice researchers with a web-interface that enables them to browse all 55 four SNP panels, use online toolkits, and retrieve the original data and scripts for a variety of 56 population genetics analyses on local computers. The SR4R is freely available to academic 57 users at http://sr4r.ic4r.org/.

Keywords: Rice; SNP; Database; Hapmap

\section{Introduction}

62 Oryza sativa, or rice, was the first crop genome to be sequenced. In the past decade, 63 thousands of rice accessions in the germplasm banks worldwide have been genotyped [1] and 64 numerous rice variation databases have been constructed. One of these databases is the rice 65 variation database (RVD), a daughter database of the Information Commons for Rice 66 consortium (IC4R) [2]. RVD is a collection of over eighteen million SNPs (single nucleotide 67 polymorphisms) identified from 5,152 rice accessions based on whole-genome resequencing 68 data, and offers an ultra-high-density rice variation map - about one SNP per twenty bases on 69 average. The information contained in this high volume of raw SNPs is not ready for use until 
70 it has been processed to remove low-quality SNPs, such as those with missing/low frequency 71 genotypes, or redundant SNPs identified due to linkage disequilibrium (LD). In addition, 72 different types of research require different magnitudes of SNPs to ensure efficient computing and accurate results; for example, the requirements are different for evolutionary studies using comparative genomics and pan-genome analysis, gene mapping by quantitative trait loci (QTL), genome-wide association study analysis (GWAS), molecular breeding by marker-assisted selection (MAS) and genomic selection (GS), and variety protection by DNA fingerprint barcoding.

Construction of a reference haplotype map (HapMap) to represent the maximal population diversity for a species is the first step. The $\sim 18$ million raw SNPs in RVD provide an initial variation dataset to generate a reference HapMap for rice. According to international human HapMap database, which contains over 3.1 million high-quality SNPs, a density of one SNP per 100 bases is sufficient for performing genotype imputation, GWAS analysis and mapping of causal variations [3]. Because the genome size of rice is $\sim 400 \mathrm{Mbp}$, about two million high-quality SNPs may offer an ideal density of one SNP per 200 bases. Such density of a reference rice HapMap is especially useful for molecular breeders to perform genotype imputation to supplement missing genotypes or increase SNP density, as low-density genotyping platforms are mostly used in rice to lower genotyping expense.

For population genetics studies in which thousands of individual samples are assessed, the millions of SNPs in an entire HapMap are excessive. The redundant SNPs in a HapMap extensively increase computing costs, and may also reduce the accuracy of results. To circumvent these challenges, a subgroup of SNPs whose genotypes significantly correlate with other SNPs in the same linkage disequilibrium (LD) region are selected; these are known as tagging SNPs. The number of tagging SNPs may vary between species and populations, depending upon the lengths of LD regions in each group [4]. Based on the data in RVD, LD length in rice ranges from 100 to $500 \mathrm{~Kb}$; thus 100,000 SNPs, which yields a density of one tagging SNP per 3 to $5 \mathrm{Kbp}$, is sufficient for various genetic diversity analysis.

The expense of genotyping is an important factor to consider in crop molecular breeding, as molecular breeding typically requires the rapid genotyping of thousands of samples, often within days or even hours. Therefore, low SNP density genotyping technologies, such as SNP chip or KASP-based platforms are usually preferred by industrial seed companies; these methods offer great flexibility by combining the rapid identification of low numbers of SNPs 
102 (several to a few dozen) with the ability to multiplex hundreds to thousands of DNA samples.

103 However, these methods lack precision.

104 Modern breeding methods demand the efficiency and stability of a highly concise marker 105 panel containing 1K SNPs. SNPs used to select plants for breeding typically occur in genes 106 or genomic regions that are associated with agronomic traits believed to be subject to selective pressures [5]. Genes with variations exhibiting selectively fixed signatures can be identified based on the $\theta \pi$ and $F s t$ values computed by selective sweep analysis [6]. This magnitude of SNPs is suitable for synthesis on low-density SNP chips, which are then used for conducting certain types of molecular analysis, such as marker-assisted selection, seed purity or heterozygosity testing, genetic component analysis, and subpopulation classification. For intellectual protection of commercial rice varieties, DNA fingerprinting typically uses only 12 to $36 \mathrm{SNPs}$, to generate a combination of barcodes with maximal resolution to distinguish commercial varieties in the seed industry or germplasm accessions in gene banks.

115 Simulation of all possible combinations of a set of candidate SNPs have to be tested in a large germplasm population to ensure the maximal resolution with fewest markers, such as the MinimalMarker algorithm [7].

To enhance the ability of researchers to effectively use the RVD in IC4R, we developed a daughter database we have called SnpReady for Rice, or SR4R. SR4R enables researcher to readily retrieve SNPs that are relevant to their own research, thus saving time and computational resources. In SR4R, the $\sim 18$ million SNPs have been divided into four categories: hapmapSNPs, tagSNPs, fixedSNPs, and barcodeSNPs (Figure 1). SR4R allows users to browse the related information associated with each SNP panel, and also to download each set of genotype files for local use. SR4R also offers 18 bioinformatics tools and pipeline scripts, enabling users to locally run the tools to perform genotype imputation, basic statistical analysis, genotype file format conversion, SNPs filtration and extraction, population structure analysis, genetic diversity analysis, rice subpopulation classification, DNA fingerprinting analysis, and other additional functions. 
132 The IC4R rice variation database (RVD; http://variation.ic4r.org/) is a collection of over 18

133 million SNPs with related annotation information, identified from previously published 134 whole genome resequencing of 5,152 rice accessions [2]. Such a high-density rice variation 135 map, which identifies an average of one SNP per twenty bases, offers the possibility of 136 generating a high-density HapMap for the rice research community; creating such a HapMap 137 was the first step in creating the SnpReady for Rice (SR4R) Database described here.

138 To ensure the quality of HapMap, we performed an initial filtration of samples and SNPs 139 on the raw dataset of 5,152 accessions (Materials and Methods). First, a total of 2,556 140 accessions with genotype missing rate less than $20 \%$ were selected; each selected accession 141 has been documented with explicit subpopulation classification and origins (Table S1). Then, 142 SNPs with genotype missing rate $\geq 0.1$ and minor allele frequency (MAF) $\leq 0.05$ were 143 removed. Genotype imputation on the resulting 2,883,623 SNPs in the selected 2,556 144 accessions yielded a high-quality HapMap containing 2,097,405 SNPs without any missing 145 genotypes using the software Beagle [8]. These 2,097,405 SNPs were regarded as the 146 hapmapSNP panel, and were used as the initial dataset for generating the other three SNP panels (Figure 2A and 2D).

The generated reference HapMap of rice has an average density of five SNPs per Kb with a heterozygosity rate of $3.75 \%$ (Figure 2B and 2D). Genome-wide distribution statistics showed that $58.4 \%$ of the hapmapSNPs present in the intergenic regions, $12.5 \%$ in the intronic regions, $11.8 \%$ in the exonic regions, $0.02 \%$ on the splicing sites, and $10.6 \%$ and $6.8 \%$ hapmapSNPs located in the upstream and downstream regions ( $1 \mathrm{~Kb}$ away from transcription start site or transcription end site) of a gene territory (Figure 2F). The 2,097,405 hapmapSNPs with genotypes of 2,556 accessions are available to download, enabling users to perform genotype imputation on local genotype data to increase the density of SNPs generated from low-density genotyping platform.

\section{The tagSNP panel}

159 High SNP density is usually beneficial to precise mapping of trait-related genes with GWAS 160 analysis, but is not suitable for population genetic analysis because SNP redundancy may add 161 unnecessary computation costs and introduce bias to the results [9]. Since SNPs within the 162 same LD region possess correlated genotypes forming one haplotype block, a representative 
163 SNP is usually selected as a tag to solve the redundancy issue. We adopted an LD-based SNP

164 pruning procedure to infer haplotype tagging SNPs (tagSNPs) from the hapmapSNPs 165 (Materials and Methods). As a result, 156,502 tagSNPs were identified (Figure 1). To 166 verify whether the tagSNP panel properly represents the genetic diversity of the population, 167 phylogenetic analysis using the 156,502 tagSNPs was performed on the 2,556 rice accessions 168 which were explicitly documented with subpopulation classification and origins. As shown in 169 Figure 3A, the resulting phylogenetic tree clearly exhibited six major clades representing the 170 five cultivated rice subpopulations and one wild rice subpopulation. The five cultivated rice 171 subpopulations include indica rice (Ind for short) containing 1,655 accessions, Aus rice (Aus) 172 containing 182 accessions, Aromatic (Aro) rice containing 56 accessions, tropical japonica 173 rice $(\operatorname{Tr} J)$ containing 318 accessions, and temperate japonica rice (Tej) containing 327 174 accessions, whilst the wild rice subpopulation contains 18 O. rufipogon (Oru) accessions. In 175 addition, PCA-based (Figure 3B) and admixture-based (Figure 3C) analyses showed the 176 same pattern, with the subpopulation classification as the phylogenetic tree indicated. For 177 population admixture structure analysis, a predefined parameter of " $\mathrm{K}$ value" was used to 178 mandatorily estimate the number of ancestral subpopulation and uses different colours for 179 each $\mathrm{K}$ value to represent the number of subpopulations. Because the optimal number of 180 ancestral subpopulation is usually unknown, a common way is to use a series of $\mathrm{K}$ value to 181 estimate the optimal K parameter. It is worth noting that the japonica, indica and Aus subpopulations were explicitly separated when $\mathrm{K}$ was set to 3 , while the six subpopulations were clearly separated until the $K$ value was set to 8 . In addition, between $K=4$ to 7 , the indica subpopulation showed clear structure divided into six groups (indica g1 to g6) as indicated by both PCA and admixture analysis (Figure 3D and Figure S1). The genetic structures of the six rice subpopulations and the six indica subgroups are consistent with multiple previous reports [10].

\section{Genetic diversity analysis with the tagSNP panel}

The tagSNP panel represents a subset of the hapmapSNPs after approximately $92.5 \%$ of the genetic redundancy was removed (Figure 1). To test the effectiveness of the 156,502 tagSNPs, we performed another series of standard genetic diversity analyses and examined whether the results agreed with previously reported conclusions. First, we found that the count of homozygous SNPs and the heterozygosity rate of the accessions in the six 
195 subpopulations showed opposite trends: while the accessions in the TeJ subpopulation had

196 the highest count of homozygous SNPs and lowest heterozygous rate, the accessions in the 197 indica subpopulation had the lowest count of homozygosity SNPs and highest homozygosity 198 rate (Figure 4A and 4B). The IBS (identity by state) analysis is a commonly used method to 199 measure the similarity of alleles in a designated population, which may reflect the genetic 200 diversity of the whole population and subpopulations. Comparison of the IBS values among 201 different subpopulations may help understand the degree of genetic differentiation in 202 different subpopulations. In order to validate whether the IBS results generated from the 203 tagSNPs are consistent with the previous reports regarding the genetic diversity in different 204 subpopulations, pairwise computation of the IBS values between each pair of accessions 205 within the same subpopulation was performed, and the results showed that temperate 206 japonica rice has the highest IBS values, while the indica rice has the lowest (Figure 4C). In 207 addition, runs of homozygosity $(\mathrm{ROH})$ analysis indicated that the temperate japonica rice has 208 the most and longest $\mathrm{ROH}$ regions, while the indica rice has the least and shortest $\mathrm{ROH}$ 209 regions (Figure 4D). This pattern agreed with the result from LD decay analysis showing that 210 temperate japonica rice has the slowest LD decay rate while the indica rice has fastest rate 211 (Figure 4E). Computations of $\theta \pi$ and $F s t$ are commonly used methods to measure genetic 212 diversity within population and between population, respectively (Materials and Methods).

213 The within-subpopulation diversities of the six rice subpopulations are Oru $(\theta \pi=0.218)$, Ind $214(\theta \pi=0.216)$, Aus $(\theta \pi=0.182)$, Aro $(\theta \pi=0.145), \operatorname{Tr} J(\theta \pi=0.116)$ and $\operatorname{TeJ}(\theta \pi=0.068)$. Using the 215 wild rice subpopulation as reference, the genetic distances of the five types of cultivated rice 216 between wild rice are $T e J(F s t=0.476), \operatorname{TrJ}(F s t=0.419)$, Aus $(F s t=0.299)$, Ind $(F s t=0.266)$ 217 and Aro ( $F s t=0.241$ ), suggesting the highest domestication level of japonica rice compared to 218 other rice (Figure 4F and 4G). The collective results from multiple angles of standard 219 genetic diversity analyses were consistent with previous reports that indica rice has a more 220 complicated genetic composition and origin compared to the other five subpopulations [11].

\section{Genomic selection analysis with the tagSNP panel}

223 Genomic selection (GS) has been widely used in industrial animal and crop breeding 224 programs [12]. GS is essentially a best linear unbiased prediction (BLUP) model that is first 225 trained with known genotypes and phenotypes of reference population individuals, usually 226 accounting for $20 \%$ to $50 \%$ of a breeding population, and then used to predict the unknown 
227 phenotypes of the remaining genotyped individuals (the candidate population). The predicted 228 phenotypes, known as the genomic estimated breeding values (GEBV), are ranked from high 229 to low, and can be used to assist in deciding upon a hybridization plan. Although GS may 230 significantly shorten the breeding cycle, the cost for genotyping has been a vital factor 231 because the GS model has to take genome-wide SNP markers as input, especially from crop 232 breeding in which thousands to hundreds of thousands of individuals need to be genotyped. 233 In order to lower genotyping cost, compilation of a set of thousands of SNPs that may best 234 represent the overall genetic backgrounds of a breeding population is of great importance.

235 Because the 156,502 tagSNPs category is a high-quality marker set with most redundancy 236 removed while preserving maximal genetic diversity, it may be considered as a marker pool 237 for selecting high-efficiency SNPs for genomic selection. To test the effectiveness, we 238 analysed a previously published dataset containing 414 rice parental lines with non-missing 239 genotypes of 29,434 SNPs profiled by the $44 \mathrm{~K}$ rice SNP chip, and nine phenotype traits 240 (flowering time, panicle fertility, seed width, seed volume, seed surface area, plant height, 241 flag leaf length, flag leaf width, and florets per panicle). The GS model was obtained from 242 the ridge regression best linear unbiased prediction (rrBLUP) algorithm [13], and prediction 243 accuracy was evaluated with Pearson correlation between observed and predicted traits by 244 five-fold cross validation. The evaluation was performed using five different SNP 245 combinations: Set-1, the original 29,434 SNPs on the 44K chip; Set-2, the 1,090 SNPs 246 overlapped between the 156,502 tagSNPs and 29,434 SNPs; Set-3, the 1,090 SNPs randomly 247 selected from the 29,434 SNPs; Set-4, the 1,090 SNPs evenly distributed in the genome (350 $248 \mathrm{~Kb}$ per SNP) selected from the 29,434 SNPs; and Set-5, the 1,090 consecutive SNPs 249 localized within a randomly selected genomic region from the 29,434 SNPs. Then the 250 rrBLUP prediction was performed on the nine phenotypes using the five sets of SNPs to 251 compare prediction accuracies (Figure 5). Although prediction accuracies greatly varied 252 ranging from 0.23 to 0.90 among the nine traits due to different heritability of each trait, the 253 trend of the five SNP sets within the same trait was generally consistent. Except for the trait 254 of panicle fertility in which the Set-2 SNPs exhibited the highest prediction accuracy, the full 25529,434 SNPs showed the highest prediction accuracy for the other eight traits followed by the 2561,090 tagSNPs in the second position. We further performed pairwise student's t-test for 257 Pearson correlations of the selected 1,090 tagSNPs set (Set-2) and other four sets, the result 258 shows that the selected 1,090 tagSNPs set significantly outperform other randomly selected 259 SNP set for most traits (Figure S2). These results indicate that selection of about one 
260 thousand tagSNPs from the tagSNP pool might be a feasible option to lower genotyping

261 budget; for example, these SNPs could inform the synthesis of a new low-density SNP chip 262 rather than using high-density SNP chip.

\section{The fixedSNP panel}

265 In the crop breeding industry, genotyping cost-per-sample is a top-priority factor, since 266 hundreds to thousands of samples are often genotyped in single day. The data then assists a 267 variety of molecular breeding practices, including genomic selection-assisted phenotype 268 prediction, marker-assisted backcrossing, seed purity or genotype heterozygosity analysis, 269 and subpopulation identification. Cost reduction is usually fulfilled by compiling a highly 270 effective marker panel containing only dozens to hundreds of SNPs that are available for 271 high-throughput genotyping platforms, such as Douglas ArrayTape and LGC Omega-F 272 equipment, using a PCR-based $\mathrm{KASP}^{\mathrm{TM}}$ genotyping assay. These systems allow users to 273 flexibly combine different numbers of SNPs and DNA samples using multiple plates with 96 274 and 384 wells per run. To meet the industrial demand, further compression of the tagSNP 275 panel must consider not only the genetic relationship between subpopulations and accessions, 276 but also the evolutionary and/or functional significance of SNPs with high diagnostic 277 effectiveness and stability.

278 The $F s t$ and $\theta \pi$ values are commonly used indicators of genomic regions demonstrating 279 signatures of selective sweeps, caused by domestications, artificial selections and 280 environmental adaption. SNPs in selective-sweep regions are usually evolutionarily fixed 281 with strong positive selection signals. To generate the fixedSNP panel, we first identified the 282 selective sweep regions that are specific to each subpopulation and are common to the six 283 subpopulations by combining the ratio of $F s t$ versus $\theta \pi$ based on the comparison of the 284 cultivated subpopulation against the wild rice population (Materials and Methods). Using $285100 \mathrm{~Kb}$ and $10 \mathrm{~Kb}$ windows, large and small genomic regions showing selective sweep 286 signals were identified, respectively. In total, 227 (cultivated vs. wild), 381 (Ind vs. wild), 333 287 (Aus vs. wild), 296 (Aro vs. wild), 256 (TrJ vs. wild) and 269 (TeJ vs. wild) identified regions 288 showed significantly smaller Tajima' D values compared to other regions (Figure 6A). 289 Subsequently, genes located in the selective sweep regions and their corresponding GSEA 290 (Gene Set Enrichment Analysis) terms were further identified for each subpopulation, and $291 \sim 50 \%$ of them were specific to each subpopulation whilst only 27 GSEA terms co-exist in the 
292 five cultivated rice subpopulations (Figure 6B). Finally, a total of 1,180 SNPs occurred within the genes in the selective sweep regions were selected to generate the fixedSNP panel.

\section{Subpopulation classification analysis with the fixedSNP panel}

296 To evaluate the fixedSNP panel, subpopulation classification with phylogenetic tree analysis was performed using the 1,180 fixedSNPs, and the results were compared to the results generated from the 156,502 tagSNPs performed on the same population of 2,556 accessions. All of the accessions were assigned to the correct subpopulations with tagSNPs and the phylogenetic tree showed consistent structure with the tree constructed with fixedSNPs (Figure 6C). To further evaluate the universality of the fixedSNP panel, we performed subpopulation classification on two external populations genotyped by SNP chips [11] [14]. One chip dataset contained 880 cultivated rice accessions genotyped by the Affymetrix $700 \mathrm{~K}$ SNP chip, while the other contained 351 cultivated accessions genotyped by the Illumina 44K SNP chip. Both external chip datasets have been documented with clear subpopulation classification and origins, and possess relatively high genetic diversity. Only 314 and 63 SNPs from the $700 \mathrm{~K}$ and $44 \mathrm{~K}$ chips, respectively, were found in the 1,180 fixedSNP panel. For the chip dataset containing 880 accessions, 877 accessions were correctly assigned to their documented subpopulations; three TeJ accessions (IRGC121549, IRGC121520 and IRGC121535) were incorrectly assigned to the $T r J$ subpopulation (Figure 6D). As for chip dataset containing 351 accessions, 348 were assigned to the correct subpopulation; three TeJ accessions (NSFTV134, NSFTV204 and NSFTV283) were mistakenly assigned to Trj rice (Figure 6E). Overall, $99.8 \%$ of the rice accessions examined were assigned to previously documented subpopulation records using markers extracted from the fixedSNP panel, indicating that the fixedSNP panel is an efficient, accurate new tool for subpopulation classification.

\section{The barcodeSNP panel}

DNA fingerprinting technology using a small set of SNPs to generate a series of genotype combinations, referred to as "barcodes," has become an economical means to protect commercialized varieties. Thus, the barcodeSNP panel must be able to uniquely identify these barcodes to distinguish between each of the rice varieties on the market. To ensure 
323 highest uniqueness but lowest count of barcodeSNPs, we applied the MinimalMarker

324 algorithm on the fixedSNP panel to exhaustively traverse all possible genotype combinations 325 that would distinguish the 2,556 accessions (Materials and Methods). The MinimalMarker 326 algorithm generate three sets of minimum marker combinations, in which each set contains 32728 SNPs. After merging the three sets, 38 barcodeSNPs were finally selected to generate the 328 panel (Figure S2A). In addition, up- and down-stream flanking sequences were also provided 329 for users to design primers for PCR-based KASP ${ }^{\mathrm{TM}}$ genotyping assays. The SR4R also offers 330 a web interface that allows users to identify corresponding accessions or varieties when rice 331 varieties are submitted for genotyping with any number of barcodeSNPs between 8 to 38 . 332 The SR4R returns a list of the top 10 best-matched accessions/variety in the database, and 333 displays associated information including the accession/variety IDs, number of mismatched 334 bases, genomic position of the barcode, genotype heterozygosity, and documented 335 subpopulation and origin. Among the top 10 hits, if multiple best-matched varieties with $336100 \%$ identity are returned using a certain number of barcodes, the users may genotype 337 additional barcodeSNPs until a unique best matched variety is identified. It is worth noting 338 that because the SR4R does not have a complete list of the barcodes for all commercial rice 339 varieties in the database, the 38 barcodeSNPs is considered as an initial panel for users to test 340 the best combinations with the most optimal sensitivity and specificity using flexible numbers 341 of markers.

\section{Machine learning analysis with the barcodeSNP panel}

344 If a new variety genotyped with barcodeSNPs is not found in the database, SR4R will 345 perform subpopulation classification. The traditional method of subpopulation classification 346 first integrates the genotype of the submitted variety with the genotypes of all the varieties in 347 the database, then performs phylogenetic analyses to determine the best assigned 348 subpopulation. This procedure is tedious and computationally inefficient since the database 349 contains hundreds of thousands of accessions. To simplify the procedure so that it may be 350 implemented through a web interface, we adopted an alternative method that utilizes machine 351 learning-based subpopulation classification models with the 38 barcodeSNPs as features. We 352 used all of the 2,556 rice accessions to evaluated seven commonly used machine learning 353 algorithms to perform subpopulation classification including decision tree, k-nearest 354 neighbouring, naïve Bayesian, artificial neural network, random forest, multinomial logistic 
355 regression and one-vs-rest logistic regression algorithms, followed by ten-fold cross

356 validation assessment (Materials and Methods). A series of assessments of the classification 357 precision in the five cultivated rice subpopulations indicated that, out of the seven methods 358 the best one is the multinomial logistic regression model, whose AUC (Area under curve) 359 values were all $\geq 0.99$ for all subpopulations (Figure S3B-F). Additional methods are one-vs360 rest logistic regression and the random forest model; where results from each yielded similar 361 classification precision to the multinomial logistic regression model. Then, we used an 362 independent datasets containing 880 rice accessions profiled by $770 \mathrm{~Kb}$ rice SNP chip for 363 independent validation. The multinomial logistic regression model was trained by the 2,556 364 rice accessions, and then predict the subpopulation classifications on the 880 samples. The 365 AUC values were all $\geq 0.99$ for all subpopulations in this independent datasets, indicating 366 robustness of the model. Moreover, compared the original label and the predicted label with 367 the max probability for each sample, the true positive rate (TPR) and false positive rete (FPR) 368 are also reasonable (Figure S4). The pre-trained classification models with the seven 369 machine learning algorithms have been implemented on the SR4R server provided as a web 370 tool for users to perform subpopulation classification when the genotype information of the 37138 barcodeSNPs in submitted.

\section{The barcodeInDel panel}

374 InDel (Insertion and Deletion) is another form of genomic variations (usually less than $50 \mathrm{bp}$ 375 in length) that can be used as molecular markers for a variety of population analysis. From 376 the 5,152 rice accessions, a total of 4,217,174 raw InDel variations were identified using the 377 IC4R variation calling pipeline [2]. After filtering low-quality InDels, 109,898 highconfidence InDels were retained with missing rate less than 0.01 and MAF $\geq 0.05$ within 2,556 rice accessions. Among the 109,898 high-confidence InDels, we further identified 62 subpopulation-specific InDels which can be used as barcodeInDels to differentiate the six rice subpopulation TeJ, TrJ, Aro, Aus, Ind and Oru, and the six subgroups of indica rice S1-S6 (Table S3). The 109,898 high-confidence InDels can be download from SR4R for users' customized analysis. 
386 Using unified bioinformatics pipelines, the genotype data of the 18 million raw SNPs

387 identified from 5,152 rice accessions were processed to construct four reference panels of 388 SNPs for different utilizations. Because genotype data processing is a complicated and 389 computationally intensive procedure, the four SNP panels are readily usable for a variety of 390 analyses simplify task for rice researchers. For better sharing of SNPs and improvement of 391 the rice variation map utility, we developed the SnpReady for Rice (SR4R) database. 392 Through the SR4R web interface, users may directly browse the four panels and retrieve 393 detailed information related to the 2,097,405 hapmapSNPs, 125,502 tagSNPs, 1,180 394 fixedSNPs, 38 barcodeSNPs. In addition, the protein-coding genes exhibiting strong selection 395 signatures, associated with the 1,180 fixedSNPs were also included in the SR4R database 396 with detailed functional annotations (Figure 7A). When users retrieve a SNP such as the first 397 SNP "OSA01S00001362", the genomic location and the adjacent gene or the gene containing 398 the queried SNP are displayed. Users may also retrieve a visualized allele frequency map in 399 the six major subpopulations, and the six subgroups of indica rice (Figure 7B).

The users may also download the four panels of SNPs along with the original genotype files for local analysis via http://sr4r.ic4r.org/download. In addition, the "Tools" module presents 18 handy scripts and pipelines that users may install on their local computers for a variety of analysis, including basic genotype processing, population diversity analysis, rice variety identification and subpopulation classification. For example, assuming one user may want to perform a genotype imputation of $44 \mathrm{~K}$ SNP rice Chip, she or he may first download the file "hapmapSNPs-genotype.tar.gz (892 MB)" containing the genotypes of the 2,097,405 hapmapSNPs in 2,516 rice accessions. Then, the user may use the pipeline and scripts demonstrated in Figure 7C to perform imputation on a local server. SR4R also offers two modules of online analysis. The first module is to use a machine learning-based method to assign the subpopulation type based on the user-submitted genotype file including no more than 20 samples. The model will return the probability of the type of subpopulation assigned to each sample (Figure 7D). The second module is to perform DNA fingerprint analysis. When the user submits a genotype file containing no more than 20 samples, the model will search the accession database, and return the top three matches of existing varieties with the number of mismatched nucleotide and heterozygosity rate displayed (Figure 7E). The programs and scripts for these two modules along with demo input and output files are also available to download for local analysis of genotypes with large sample numbers. 


\section{Conclusions}

420 The IC4R Rice Variation Database collects over 18 million raw SNPs identified from 421 resequencing of 5,152 accessions. To meet the different demands for the rice research 422 community and breeding industry, we further generated four panels of 2,097,405 423 hapmapSNPs, 156,502 tagSNPs, 1,180 fixedSNPs and 38 barcodeSNPs with standard 424 processing pipelines and uniform analytical parameters (Table S2). The four panels of SNPs 425 can be either accessed online or downloaded for local use from the daughter database of RVD 426 - SnpReady for rice (SR4R). The hapmapSNP panel contains 2 million non-missing 427 genotypes of 2,556 accessions offers a reference HapMap for genotype imputation and high428 resolution GWAS analysis. The non-redundant 150K tagSNP panel is an ideal magnitude for 429 population genetics and evolutionary analysis for research, as well as an ideal marker pool for 430 genomic selection-assisted breeding in rice. For a breeding population with about $500 \mathrm{~F}_{1}$ 431 hybrids, 1,500 to 15,000 markers selected from the tagSNP panel can be used to build a GS 432 model, reaching a satisfactory genotype-to-phenotype prediction accuracy. The fixedSNP 433 panel with high effectiveness and stability can be regarded as a marker pool for various 434 molecular breeding practice suitable for low-budget, flexible genotyping platform, in terms of 435 subpopulation classification, seed purity analysis and genetic background analysis. The 38 436 barcodeSNPs selected by MinimalMarker algorithm is an initial marker set for generating 437 DNA fingerprints for commercial rice varieties. Along with the barcodeSNP panel, two web438 based tools, one for variety identification and another for subpopulation classification, are 439 offered in SR4R. In addition, the SR4R database also offers a series of standard pipelines 440 used to construct the four sets of SNPs, and local handy tools to perform rice varieties 441 classification, barcode development, and other types of genetic and breeding research. With 442 the incremental accumulation of population genotype data in BIGD center, these 443 bioinformatics tools can be applied to other animal or plant species such as corn, wheat, 444 soybeans, for a centralized reference HapMap and SNP panel databases for plants.

Materials and Methods

\section{Construction of hapmapSNP and tagSNP panels}

448 The raw 18 million SNPs with genotype information of 5,152 rice accessions were obtained 449 from the IC4R rice variation database (http://variation.ic4r.org). Accession filtration, SNP 
450 filtration and basic statistics of homozygous SNPs and accession heterozygosity were

451 performed using in-house scripts. Genotype imputation of missing sites and phasing were

452 performed using Beagle [8]. A SNP site with missing genotype was removed if an inferred 453 genotype with a posterior probability was smaller than 0.5. Genomic annotation of 454 hapmapSNPs was performed using ANNOVAR (version 20160201) against the rice 455 International Rice Genome Sequencing Project (IRGSP) gene annotation. Using the reported 456 LD length of rice ranging from 40 to $500 \mathrm{~Kb}$, an LD-based SNP pruning method was used to 457 construct the tagSNPs category using PLINK with -indep command [15] [16]. The PLINK 458 parameters were selected based on the variance inflation factor (VIF), which recursively 459 removed SNPs within a sliding window of 50 SNPs and a step size of 5 SNPs to shift the 460 window.

\section{Tools for subpopulation structure analysis}

463 The tagSNPs for 2,556 rice accessions were concatenated as input sequences for constructing 464 the phylogenetic tree using the neighbour joining algorithm implemented in MegaCC with 465 pairwise gap deletion and 100 bootstrap replications [17]. The output tree file for all 2,556 466 rice accessions and the subtree file of indica rice accessions were visualized in MEGA7 [18]. 467 Principal component analysis of the 2,556 rice accessions was done by flashPCA [9]. 468 Population admixture structure analysis was done by fastSTRUCTURE using the variational 469 bayesian framework, and $k=2$ to $k=8$ were set to infer the admixture of ancestors for the 470 accessions.

\section{Tools for genetic diversity analysis}

473 Genetic diversity related analyses were mostly done using PLINK [16]. Genome-wide 474 pairwise IBS calculations were performed between each pair of accessions within the same 475 subpopulation in order to deduce the genetic affinity, and an IBS pairwise distance matrix 476 was generated for each subpopulation. The ROH analysis for each subpopulation used a 477 sliding window method to scan each accession's genotype for a given population at each 478 marker position to detect homozygous segments. The parameters and thresholds applied to 479 define ROH were set as follows: a minimum ROH length of $200 \mathrm{Kbp}$ and a minimum number 480 of 1,000 consecutive SNPs included in an ROH. Correlation coefficient $\left(r^{2}\right)$ of SNPs was 
481 calculated to measure LD level for each subpopulation. The average $r^{2}$ value was calculated

482 for each length of distance from 0 to $500 \mathrm{Kbp}$, followed by drawing $\mathrm{LD}$ decay figures using 483 an R script for each subpopulation. Population diversity of rice varieties was measured by 484 two indexes: $\theta \pi$ and Fst. Nucleotide diversity $\theta \pi$ was used as a measurement of the degree of 485 genotype variability within each subpopulation, while subpopulation differentiations were 486 evaluated by the fixation index $F s t$ for each of the cultivated subpopulations against the wild 487 rice population and for the cultivated subpopulations compared to each other. Values of $\theta \pi$ 488 and Fst were calculated using sites mode implemented in VCFtools [19].

\section{Tools for genomic selection analysis}

491 Genotype and phenotype datasets of the 44k rice chip were downloaded from the Rice 492 Diversity Website (http://www.ricediversity.org/). Genotype imputation and phasing were 493 then performed using Beagle (version 3.3.2), and the site was filtered if an inferred genotype 494 with a posterior probability was smaller than 0.5 . Genomic selection analysis was performed 495 using RR-BLUP mixed model implemented in R package rrBLUP [13] for nine well496 measured traits (flowering time, panicle fertility, seed width, seed volume, seed surface area, 497 plant height, flag leaf length, flag leaf width, and florets per panicle) with five different 498 feature combinations. The prediction accuracy under each feature combination was evaluated 499 by five-fold cross-validation and Pearson correlation coefficient. An example of the process 500 is as follows: the original samples were randomly partitioned into five subsets; of the five 501 subsets, a single subset was retained as the validation data, and the remaining four subsets 502 were used as training data. This process was repeated five times, with each of the ten subsets 503 used exactly once as the validation data. The Pearson correlation coefficients of the predicted breeding values and the real phenotype values were calculated for each fold.

\section{Construction of the fixedSNP panel}

$\theta \pi$ and Tajima' D values were calculated for six rice subpopulations (TeJ, TrJ, aro, aus, ind, Oru) with a sliding-window fashion across the genome using in-house scripts. Fst values were calculated for the five cultivated subpopulations against the wild Oru subpopulation, as well as for the five cultivated subpopulations against each other. For each pairwise comparison, the intersection of the top $5 \%$ windowed $\theta \pi$ ratios (wild subpopulation $v s$. 
512 cultivated subpopulation), and the top 5\% windowed $F s t$ values correspondingly were 513 selected as strong selective sweep signals. Window sizes of both $100 \mathrm{Kbp}$ and $10 \mathrm{Kbp}$ were 514 used to detect large or small selective sweep regions, followed by merging the results as the 515 candidate selective sweep regions for each subpopulation. Tajima' D distribution was also 516 drawn for the candidate selective sweep regions against the whole genomes for each pairwise 517 comparison. Genes located within the candidate selective sweep regions were extracted for 518 each comparison, and Gene Set Enrichment Analysis (GSEA) was performed for each gene 519 listed by using PlantGSEA web tools [20]. Genic SNPs located in the candidate selective 520 sweep regions identified from the above-mentioned pairwise comparisons were merged as 521 fixedSNPs.

\section{Construction of the barcodeSNP panel}

524 The 1,180 fixedSNPs were used as the initial marker set to select the minimal number of 525 barcodeSNPs that can maximally distinguish the 2,556 rice accessions using a heuristic mode 526 implemented in MinimalMarker [7]. Three minimal sets each containing 28 SNPs were 527 generated, and after merging the three sets, 38 unique SNPs were selected as barcodeSNPs 528 for generating DNA fingerprints for each accessions.

To identify commercialized rice varieties using the combination of 38 barcodeSNPs, seven machine learning-based methods were used: decision tree, k-nearest neighboring, naïve Bayesian, artificial neural network, random forest, multinomial logistic regression, and onevs-rest logistic regression algorithms in the Python sklearn library (https://scikitlearn.org/stable/). The precision of each model was assessed using ten-fold cross-validation method. Specifically, the original sample set was randomly partitioned into ten subsets in which nine subsets were used for training model and the remaining subset was used as the testing model; this procedure was repeated ten times and an average prediction accuracy was computed from the overall performance of the tested models. Five one-hot codes (10000, 01000, 00100, 00010, 00001) to label the five subpopulations for classification using machine learning models. Then, the predicted label with the max probability was compared

540 with the original label for each sample. If the predicted label is identical with the original 541 label, the prediction result was regarded as correct. Then, the ratios of positive and negative 542 rate were computed to plot ROC curves and compute AUC values. 


\section{Construction of the barcodeInDel panel}

545 Raw InDels were identified using the IC4R variation calling pipeline from the origin 5,152 546 rice accessions [2]. Then, the InDels from the 2,556 rice accessions with high sequencing 547 coverage (depth $\geq 5$ ) presented in SR4R database were extracted using customized Python 548 scripts, followed by using VCFtools [19] to filter InDels to generate a high-confidence InDel 549 dataset, with parameters of missing rate less than 0.01 and MAF $\geq 0.05$. Finally, using 550 customized Python scripts, InDels which have the same sequence type within each 551 subpopulation were retained to generate the subpopulation-specific barcodeInDel panel.

Data availability

554 All the data is freely available and downloadable at http://sr4r.ic4r.org/.

\section{Authors' contributions}

557 XFW, SHS and ZZ conceived the project; JY and CL collected the samples; JY conducted 558 the data analysis; DZ developed the database; JY, XFW, SHS and ZZ wrote the manuscript.

\section{Competing interests}

561 The authors declare no competing interests.

\section{Acknowledgments}

564 We are grateful to a number of users for reporting bugs and providing suggestions in 565 improving SR4R. This work was supported by the National Science Foundation of China 566 [31871706], by the Department of Agriculture of Guangdong Province (2018-36), Science 567 and technology program of Guangdong Province (2019B030316006) and by The Youth 568 Innovation Promotion Association of the Chinese Academy of Sciences [2017141]. 


\section{$570 \quad$ References}

571 [1] Li Z, Fu BY, Gao YM, Wang WS, Xu JL, Zhang F, et al. The 3,000 rice genomes 572 project. GigaScience 2014;3.

[2] Zhang Z, Hu S, He H, Zhang H, Chen F, Zhao W, et al. Information Commons for Rice (IC4R). Nucleic Acids Research 2016;44:D1172-80.

Frazer KA, Ballinger DG, Cox DR, Hinds DA, Stuve LL, Gibbs RA, et al. A second generation human haplotype map of over 3.1 million SNPs. Nature 2007;449:851-61.

[4] Flint-Garcia SA, Thornsberry JM, Buckler ES. Structure of Linkage Disequilibrium in Plants. Annual Review of Plant Biology 2003;54:357-74.

[5] Nielsen R. Molecular Signatures of Natural Selection. Annual Review of Genetics 2005;39:197-218.

[6] Lam HM, Xu X, Liu X, Chen W, Yang G, Wong FL, et al. Resequencing of 31 wild and cultivated soybean genomes identifies patterns of genetic diversity and selection. Nature Genetics 2010;42:1053-9.

[7] Fujii H, Ogata T, Shimada T, Endo T, Iketani H, Shimizu T, et al. Minimal marker: An

[8] Browning SR, Browning BL. Rapid and accurate haplotype phasing and missing-data inference for whole-genome association studies by use of localized haplotype clustering. American Journal of Human Genetics 2007;81:1084-97.

[9] Abraham G, Inouye M. Fast principal component analysis of large-scale genome-wide data. PLoS ONE 2014;9.

[10] Wang W, Mauleon R, Hu Z, Chebotarov D, Tai S, Wu Z, et al. Genomic variation in 3,010 diverse accessions of Asian cultivated rice. Nature 2018;557:43-9. 
595 [11] McCouch SR, Wright MH, Tung CW, Maron LG, McNally KL, Fitzgerald M, et al. Open access resources for genome-wide association mapping in rice. Nature Communications 2016;7.

[12] Spindel J, Begum H, Akdemir D, Virk P, Collard B, Redoña E, et al. Genomic Selection and Association Mapping in Rice (Oryza sativa): Effect of Trait Genetic Architecture, Training Population Composition, Marker Number and Statistical Model on Accuracy of Rice Genomic Selection in Elite, Tropical Rice Breeding Lines. PLoS

[13] Endelman JB. Ridge Regression and Other Kernels for Genomic Selection with R

[15] Mather KA, Caicedo AL, Polato NR, Olsen KM, McCouch S, Purugganan MD. The extent of linkage disequilibrium in rice (Oryza sativa L.). Genetics 2007;177:2223-32.

[16] Chang CC, Chow CC, Tellier LCAM, Vattikuti S, Purcell SM, Lee JJ. Secondgeneration PLINK: Rising to the challenge of larger and richer datasets. GigaScience $2015 ; 4: 7$.

[17] Kumar S, Stecher G, Peterson D, Tamura K. MEGA-CC: Computing core of molecular evolutionary genetics analysis program for automated and iterative data analysis. Bioinformatics 2012;28:2685-6.

[18] Kumar S, Stecher G, Tamura K. MEGA7: Molecular Evolutionary Genetics Analysis Version 7.0 for Bigger Datasets. Molecular Biology and Evolution 2016;33:1870-4..

[19] Danecek P, Auton A, Abecasis G, Albers CA, Banks E, DePristo MA, et al. The variant call format and VCFtools. Bioinformatics 2011;27:2156-8.

[20] Yi X, Du Z, Su Z. PlantGSEA: a gene set enrichment analysis toolkit for plant community. Nucleic Acids Research 2013;41:W98-103. 


\section{Figure legends}

\section{Figure 1 An overview of the four SNP panels of the SR4R database}

625 The flow chart describes procedures on how the four SNP panels were generated.

627 Figure 2 Basic statistics of the rice hapmapSNPs after four steps of genotype processing

628 After four steps of genotype processing, a series of basic statistical analyses was performed at 629 each step to exhibit the characteristics of the SNPs, including: A. statistics of individual 630 missing rate, B. statistics of individual heterozygote rate, C. statistics of minor allele 631 frequency, D. statistics of site missing rate, and E. statistics of site heterozygote rate. F. After 632 ARNOVAR analysis to annotated the hapmapSNPs, distribution of the hapmapSNPs in 633 different genomic regions were analyzed.

635 Figure 3 Population structure analysis of the 2,556 rice accessions using tagSNPs

636 To test whether the $150 \mathrm{~K}$ tagSNPs can generate the population structures consistent with 637 previous reports, we performed a series of population structure analyses to generate: A. the 638 phylogenetic tree, B. the PCA map, C. the admixture structure of 2,556 rice accessions, D. 639 the phylogenetic tree of the six subgroups of indica rice. The tagSNPs effectively and 640 accurately classified the 2,556 rice accessions to corresponding populations.

\section{Figure 4 Genetic diversity analysis of rice accessions using tagSNPs}

643 The $150 \mathrm{~K}$ tagSNPs were used in a series of population genetic analysis to show the 644 effectiveness of tagSNPs including: A. statistics of homozygous SNPs, B. statistics of 645 individual heterozygosity, C. pairwise IBS values distribution, D. statistics of ROH regions, 646 E. LD decay analysis, in the five major rice subpopulations. F. Genetic diversity $(\theta \pi)$ and 647 population differentiation (Fst) between cultivated and wild subpopulations. G. Population 648 differentiation (Fst) of cultivated subpopulations.

\section{Figure 5 Genomic selection-based phenotype prediction using tagSNPs}

651 Nine agronomical phenotypes were predicted based on rBLUP models to evaluate the 652 effectiveness of tagSNPs. Five sets of SNPs with equal amounts were compared, including 653 Set-1: the original 29,434 SNPs on the 44K chip; Set-2: the 1,090 SNPs overlapped between 654 the 156,502 tagSNPs and 29,434 SNPs; Set-3: the 1,090 SNPs randomly selected from the 655 29,434 SNPs; Set-4: the 1,090 SNPs evenly distributed in the genome (350 Kb per SNP) 
656 selected from the 29,434 SNPs; Set-5: 1,090 SNPs localized within a genomic region from 657 the 29,434 SNPs.

\section{Figure 6 Screening and validation of FixedSNPs}

A. Distribution of $\theta \pi$ ratios (wild vs cultivar) and corresponding $F s t$ values, which are calculated in $100 \mathrm{~kb}$ windows. Data points located to the right of the vertical dashed line and to the top of the horizontal dashed line are potential strong selective sweep signals (Red points, corresponding to the $5 \%$ right tails of the empirical $\theta \pi$ ratio and $F$ st values distribution, respectively). Distribution of Tajima's D values for the potential selective sweep signals and whole genomes are shown within the plot. Other comparisons for the screening of subgroup specific selective sweep signals were not shown here, but demonstrate similar trends. B. Common and specific selective signals among cultivar subgroups (Number of genes or GSEA terms are shown out and in the brackets, respectively). C. Phylogenetic tree of 2,538 rice cultivars in fixedSNPs data set. D. Phylogenetic tree of 880 rice cultivars in $700 \mathrm{~K}$ chip data set. E. Phylogenetic tree of 351 rice cultivars in 44K chip data set.

\section{Figure 7 Representative functional modules in SR4R database}

673 A. Genes exhibiting significant selection signatures in the corresponding subpopulations are 674 listed in the "Selected Genes" module in the Browser. B. Allele frequencies in different subpopulations of the first hapmapSNP (SNPID: OSA01S00001362, associated gene: Os01g0100100, position: chr01-1362, allele: Alt-A, Ref-G). C. One example of the script and pipeline for population diversity analysis. D. The online analysis module of subpopulation classification using machine learning algorithms. E. The online analysis module of rice variety identification using the 38 barcodeSNPs.

\section{Supplementary material}

\section{Figure S1 Population structure of 1,655 varieties in indica}

683 A. PCA classification for 1,655 varieties in indica subgroup. B. Structure analysis for 1,655 684 varieties in indica subgroup.

686 Figure S2 T-test for Pearson correlations of the selected 1,090 tagSNPs set and other

\section{four SNP sets}


689 the 156,502 tagSNPs and 29,434 SNPs; Set-3: the 1,090 SNPs randomly selected from the 690 29,434 SNPs; Set-4: the 1,090 SNPs evenly distributed in the genome (350 Kb per SNP) 691 selected from the 29,434 SNPs; Set-5: 1,090 SNPs localized within a genomic region from 692 the 29,434 SNPs. Different colors present different $\mathrm{P}$ values. * $\mathrm{P}$ value $<0.05$; ** $\mathrm{P}$ value $<$ 6930.01.

695 Figure S3 BarcodeSNPs and machine learning models for the classification of rice 696 varieties

697 A. Heat-map of BarcodeSNPs of 2,538 rice cultivars (Red: A, Yellow: T, Blue: G, Green: C).

698 B. Decision model. C. KNN model. D. Multinomial logistic regression model. E. Naive 699 Bayesian model F. Neural network model. G. Random forest model. H. One-vs-rest logistic 700 regression model. AUC curves were drawn using the mean values of ten cross validations for 701 B-H.

702

Figure S4 Independent validation of the machine learning model.

A. ROC curve for the $770 \mathrm{~Kb}$ rice SNP chip dataset using the pre-build multinomial logistic regression model. B. The true positive rate (TPR) and false positive rate (FPR) statistics for each subpopulation of the $770 \mathrm{~Kb}$ rice SNP chip dataset.

Table S1 Summary of 2,556 rice accessions with subpopulation classification and origins 
bioRxiv preprint doi: https://doi.org/10.1101/2020.01.11.902999; this version posted January 14, 2020. The copyright holder for this preprint (which was not certified by peer review) is the author/funder. All rights reserved. No reuse allowed without permission.

\section{$717 \quad$ Figure 1}

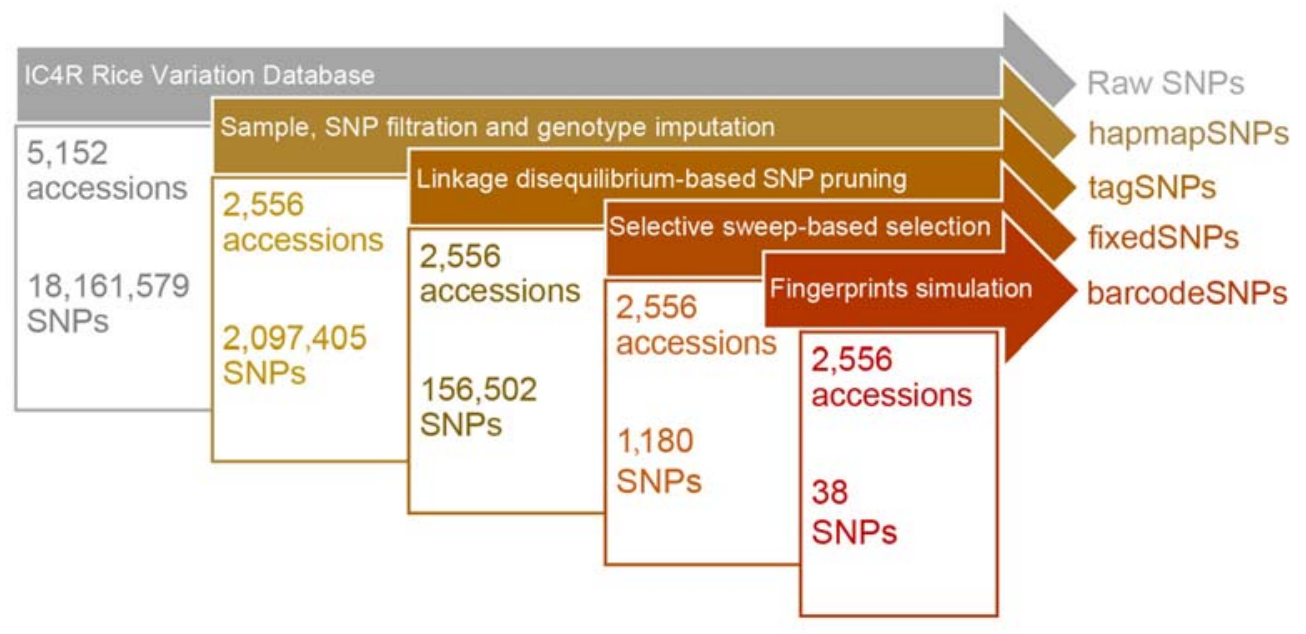

718

719

720

721

722

723

724 Figure 2

A B

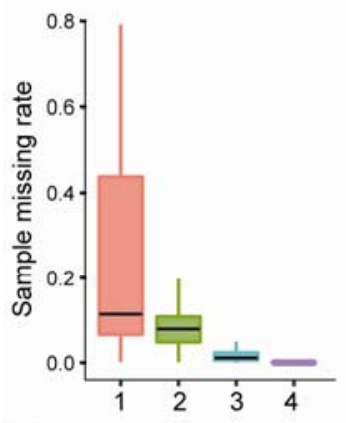

D

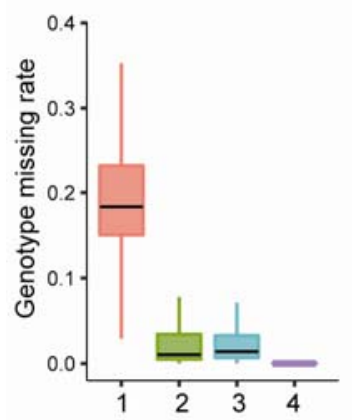

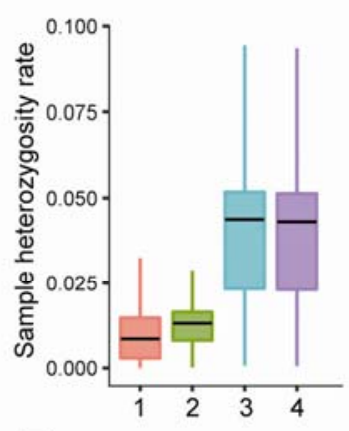

E

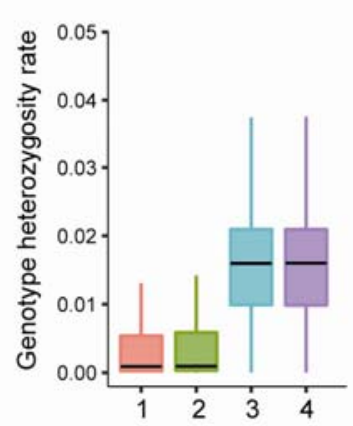

C

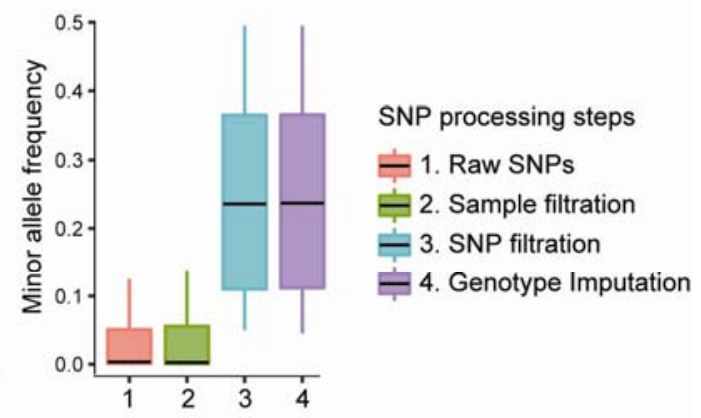

$\mathrm{F}$

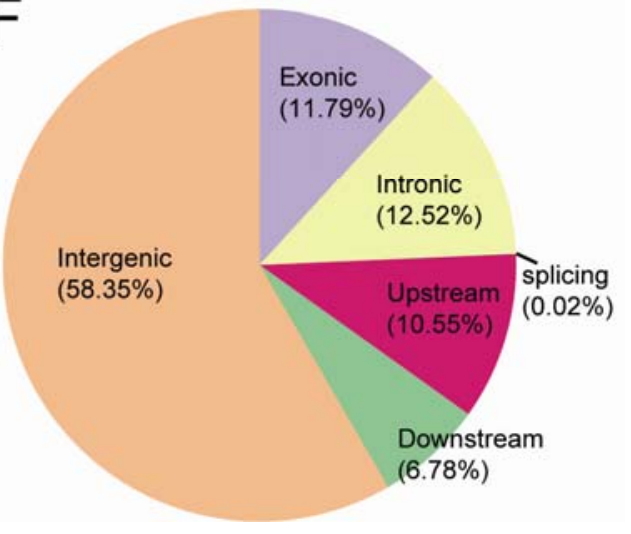


bioRxiv preprint doi: https://doi.org/10.1101/2020.01.11.902999; this version posted January 14, 2020. The copyright holder for this preprint (which was not certified by peer review) is the author/funder. All rights reserved. No reuse allowed without permission.

\section{$727 \quad$ Figure 3}
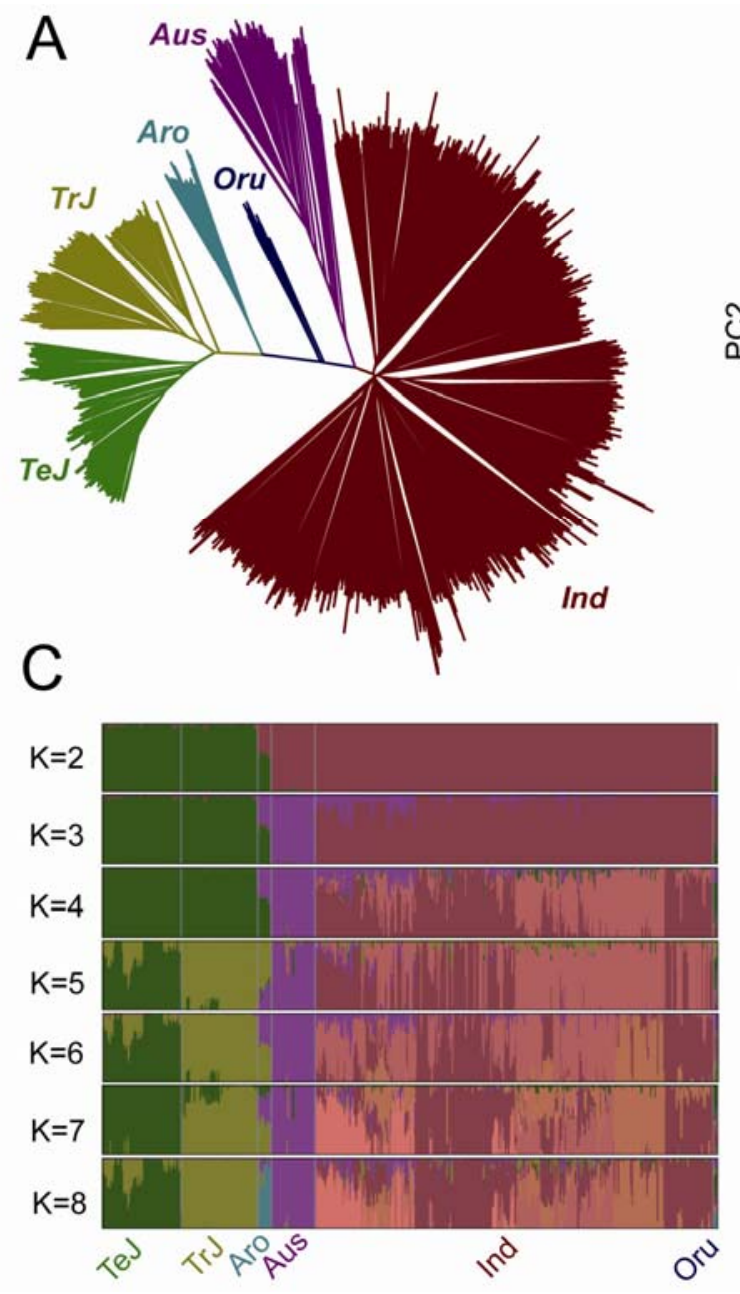

B

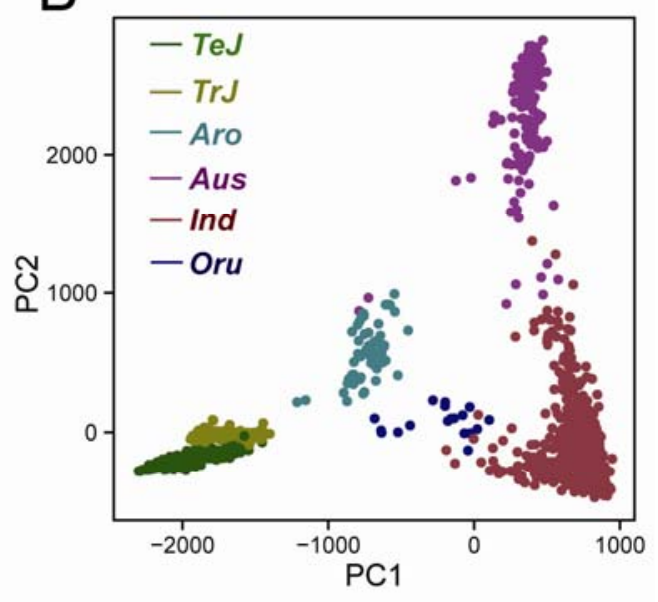

D

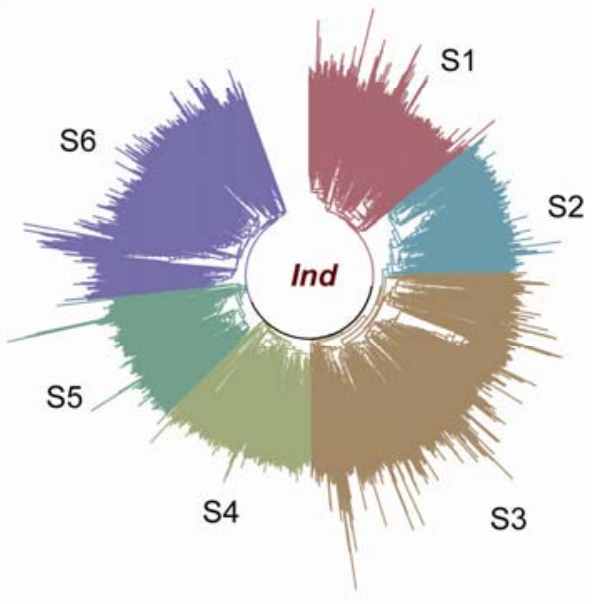


bioRxiv preprint doi: https://doi.org/10.1101/2020.01.11.902999; this version posted January 14, 2020. The copyright holder for this preprint (which was not certified by peer review) is the author/funder. All rights reserved. No reuse allowed without permission.

742 Figure 4
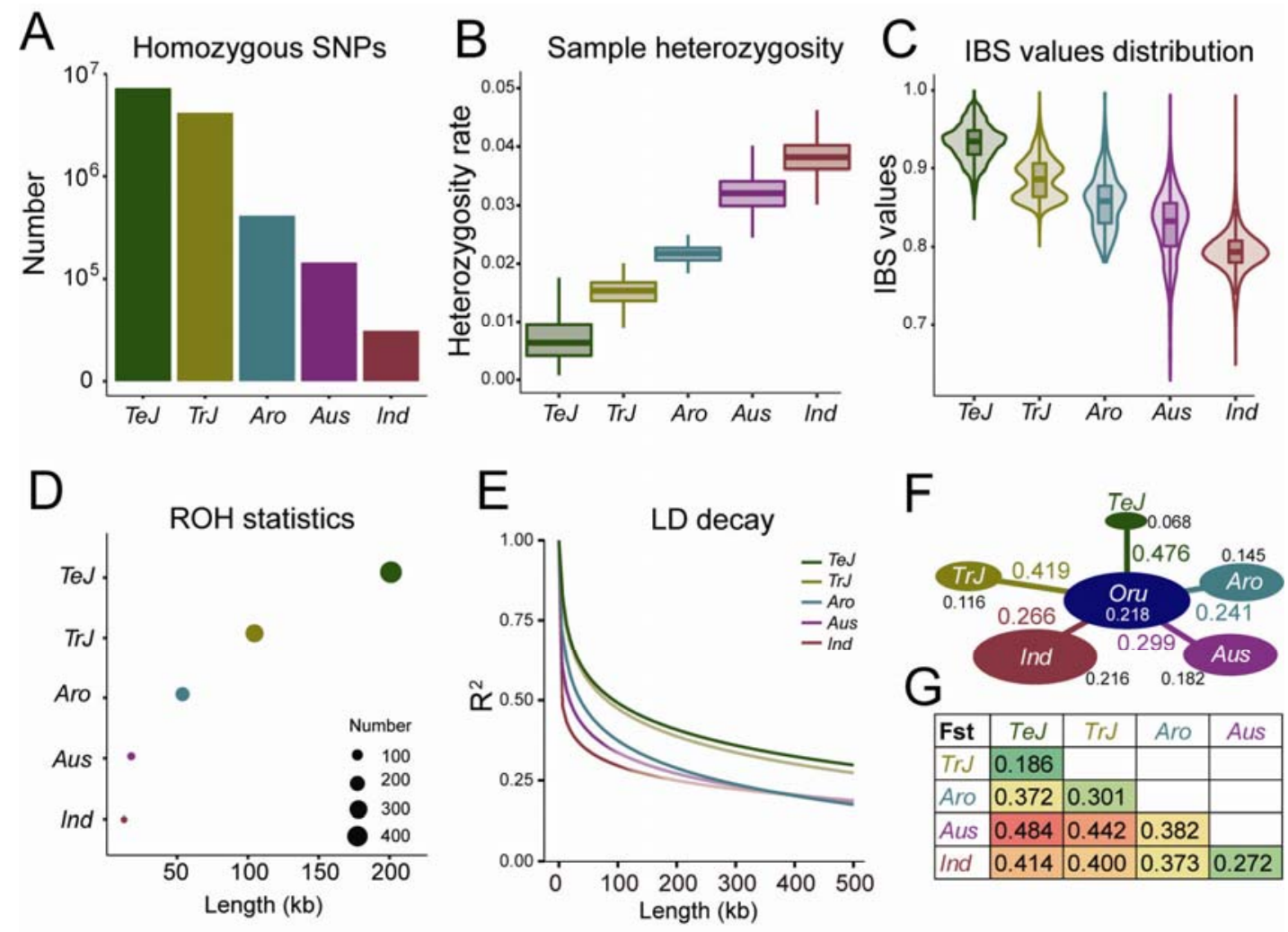

743 


\section{$760 \quad$ Figure 5}

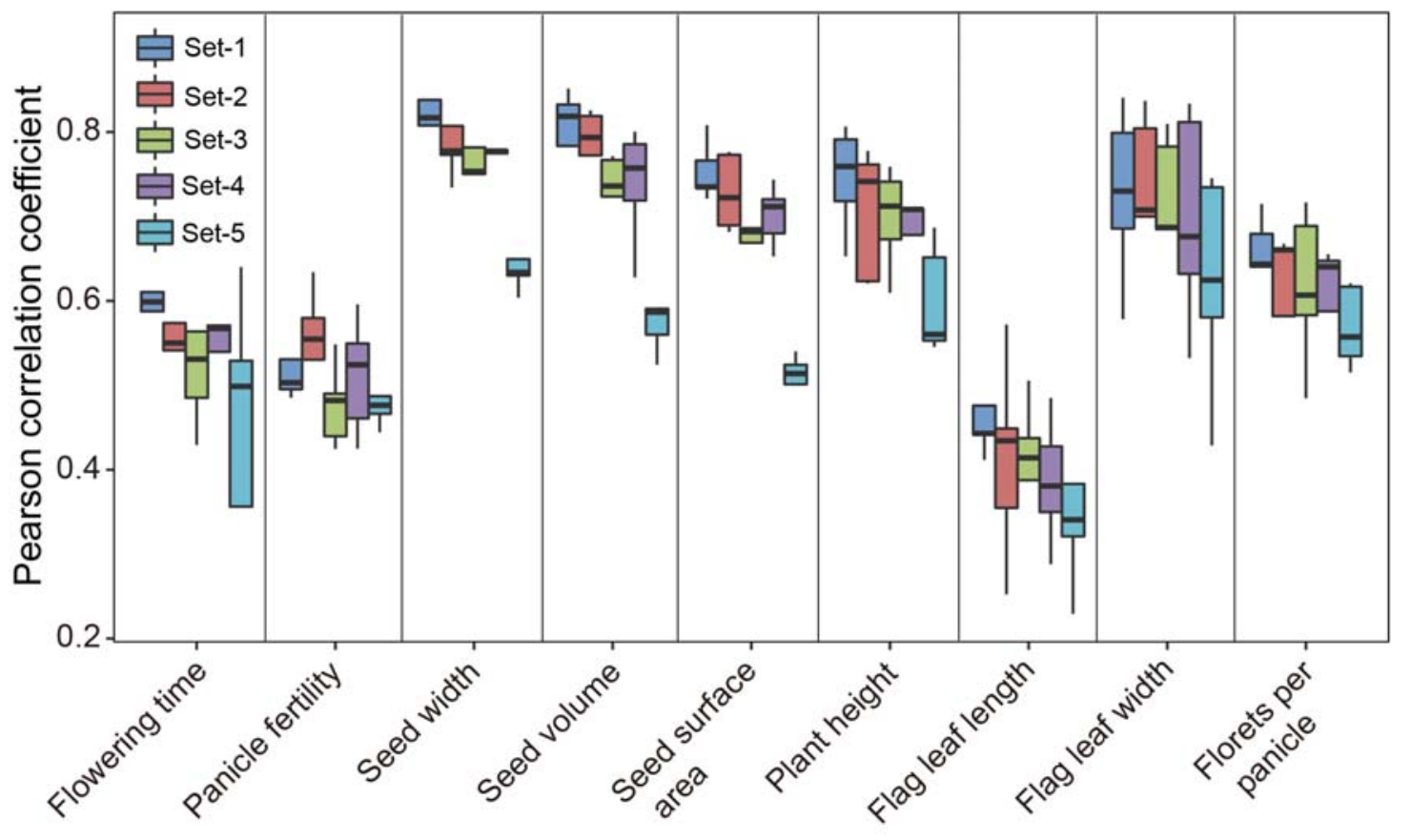




\section{$781 \quad$ Figure 6}
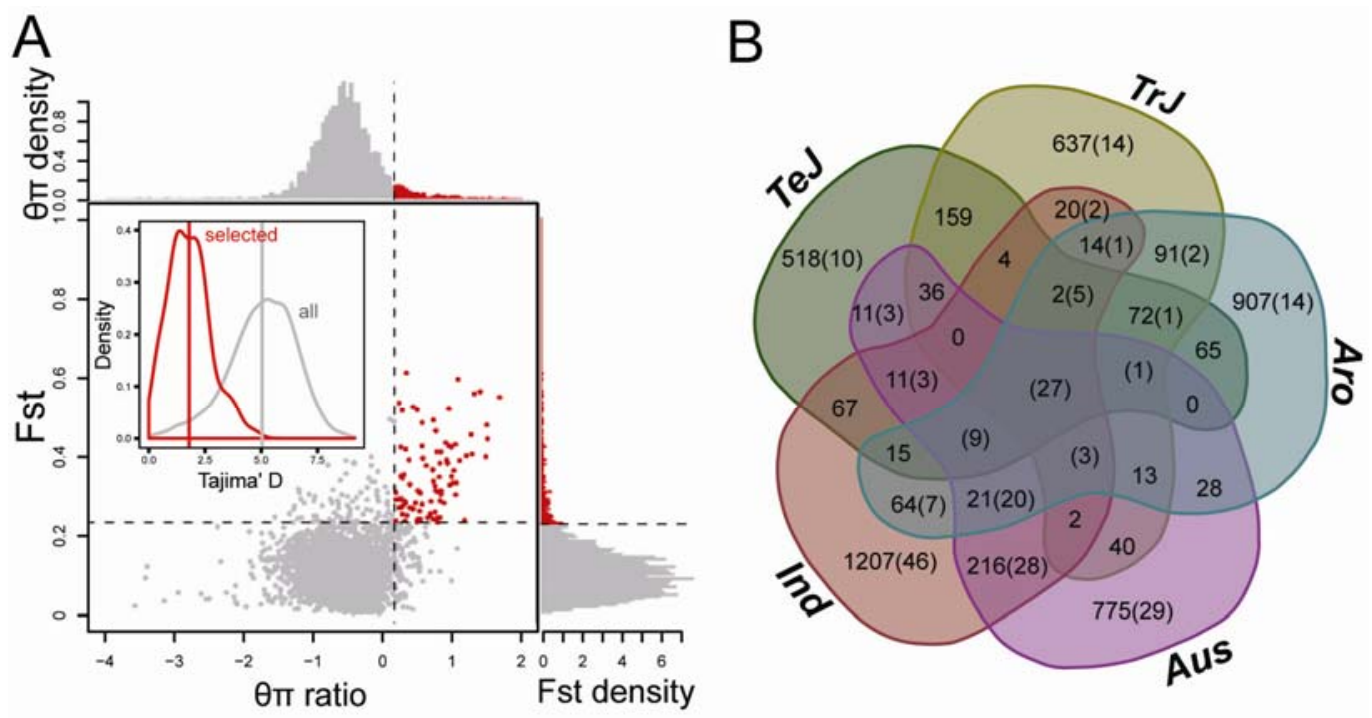

782
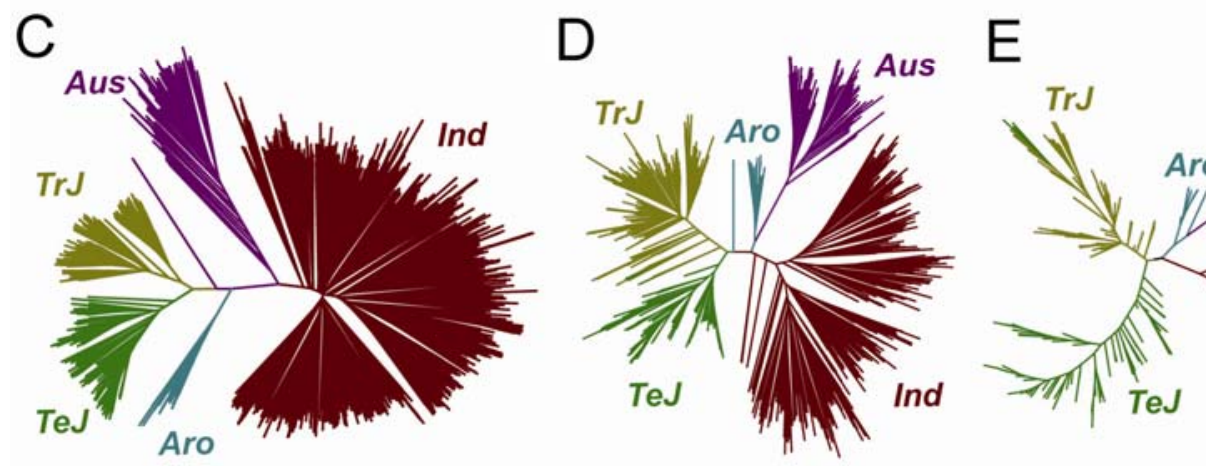

Aus

783

784

785

786

787

788

789

790

791

792

793

794

795

796 
bioRxiv preprint doi: https://doi.org/10.1101/2020.01.11.902999; this version posted January 14, 2020. The copyright holder for this preprint (which was not certified by peer review) is the author/funder. All rights reserved. No reuse allowed without permission.

\section{$797 \quad$ Figure 7}

\section{A}

\begin{tabular}{|c|c|c|c|c|c|c|c|}
\hline $\begin{array}{l}\text { Gene } \\
\text { Accession i }\end{array}$ & TeJ 1 & TrJ ${ }^{*}$ & Aro & Aus 1 & Ind & $\begin{array}{l}\text { All } \\
\text { Cultivars }\end{array}$ & Description \\
\hline Os01g0105700 & false & false & false & false & false & true & $\begin{array}{l}\text { Basic helix-loop-helix dimerisation region bHLH domain containing protein. (Os01t0105700- } \\
\text { 01)[OsbHLH071] }\end{array}$ \\
\hline Os0190105800 & false & false & false & false & false & true & Similar to Iron sulfur assembly protein 1. (Os01t0105800-01)[ISC9; OsISC9] \\
\hline Os01g0121500 & false & false & false & false & false & true & Conserved hypothetical protein. (Os01t0121500-01) \\
\hline Os01g0127450 & falso & false & false & false & false & true & $\begin{array}{l}\text { Similar to MYBL2 (ARABIDOPSIS MYB-LIKE 2); DNA binding / transcription factor. } \\
\text { (Os01t0127450-00) }\end{array}$ \\
\hline Os01g0127500 & false & false & false & false & false & true & NAD(P)-binding domain containing protein. (Os01t0127500-01) \\
\hline Os01g0127600 & false & false & false & false & false & true & Similar to Bowman-Birk type proteinase inhibitor D-\|ll precursor (IV). (Os01t0127600-01) \\
\hline Os01g0129600 & false & false & false & false & false & true & Similar to LBD40 (LOB DOMAIN-CONTAINING PROTEIN 40). (Os0110129600-00) \\
\hline Os01g0134700 & false & false & false & false & true & true & Calmodulin binding protein-like family protein. (Os01t0134700-01) \\
\hline Os01g0134800 & false & false & false & false & true & true & $\begin{array}{l}\text { Similar to (1,4)-beta-xylan endohydrolase, isoenzyme X-1I (EC 3.2.1.8) (Fragment). } \\
\text { (Os01t0134800-01) }\end{array}$ \\
\hline Os01g0134850 & false & false & false & false & true & true & Hypothetical protein. (Os01t0134850-00) \\
\hline
\end{tabular}

\section{B $\quad$ C}
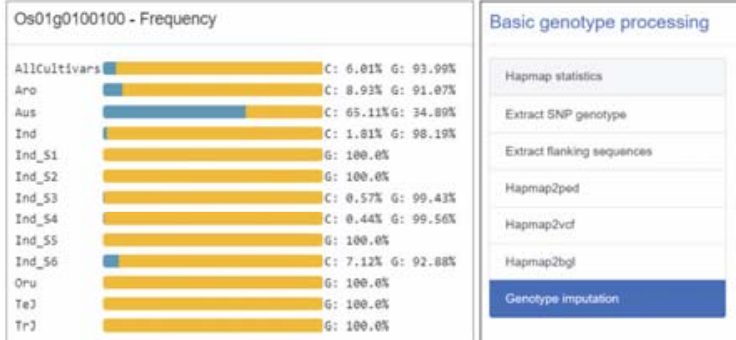

Description:

Usage:

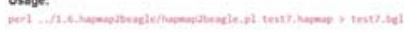

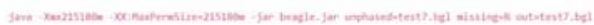

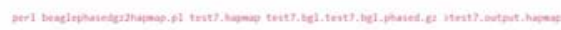

inpot tile:

Output files:

Output nies:

Parameters:

D $\quad E$
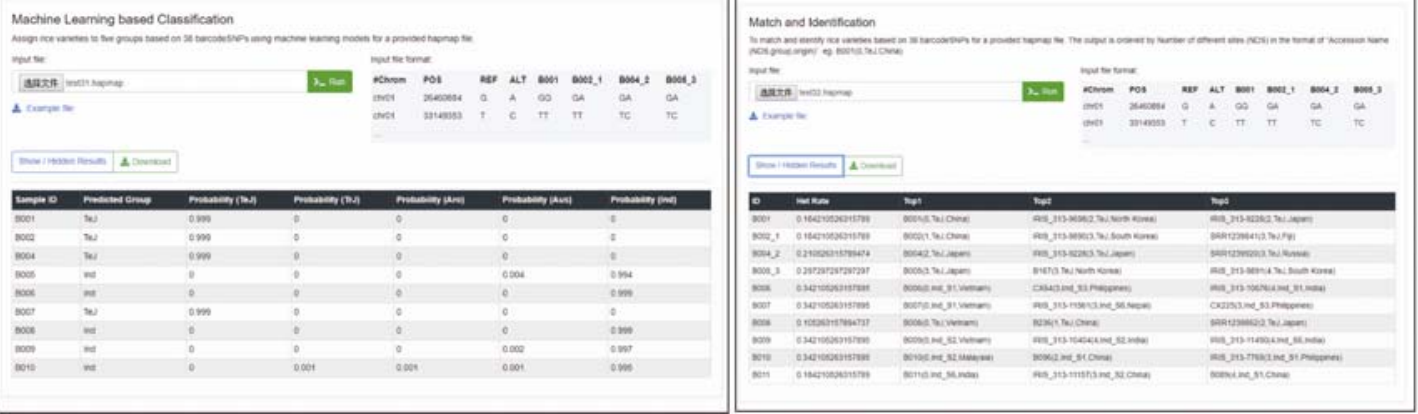

799

800

801

802 
$804 \quad$ Figure $\mathbf{S 1}$
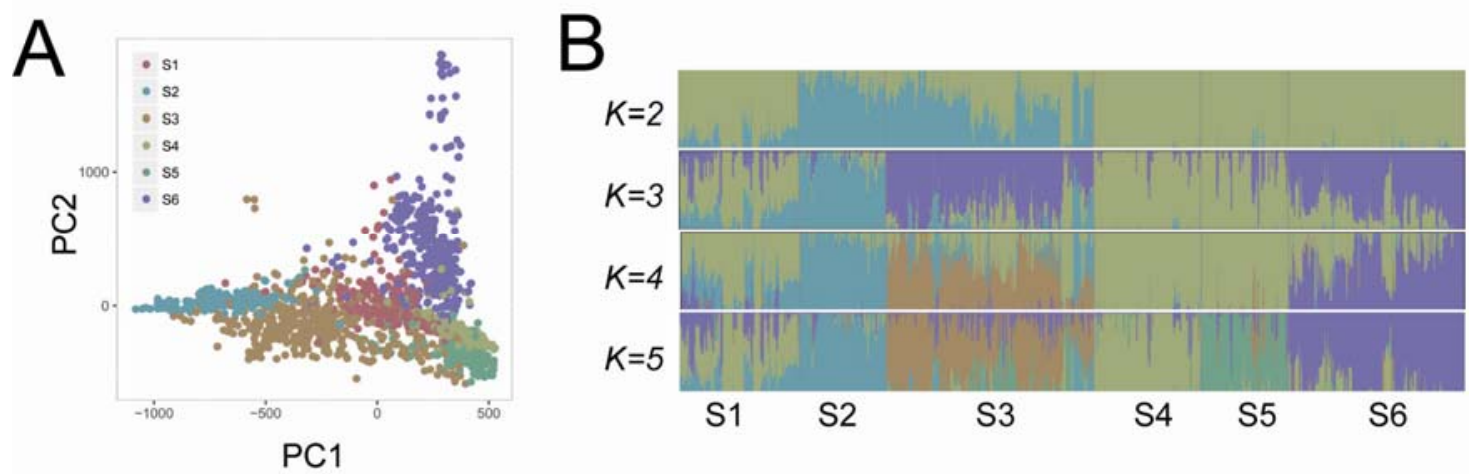

805

806

807

808

809

810

811

Figure S2

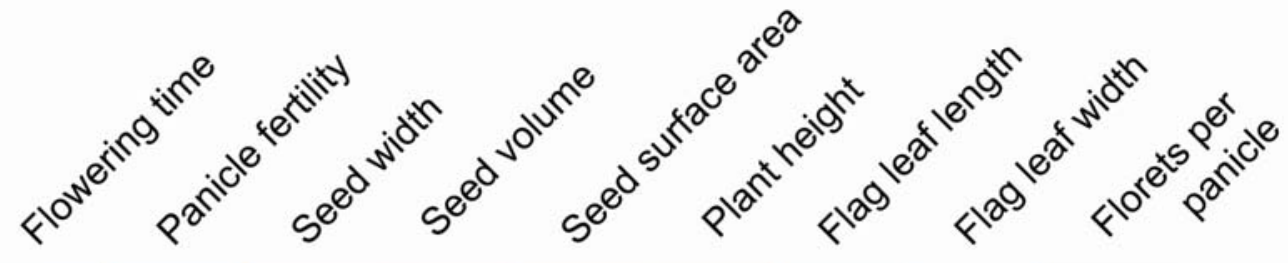

$\begin{array}{lllllllllll}\text { Set-1 vs Set-2 } & 0.2279 & 0.7783 & 0.0265 & 0.0243 & 0.0327 & 0.0346 & 0.1081 & 0.1834 & 0.0621\end{array}$

$\begin{array}{llllllllll}\text { Set-2 vs Set-3 } & 0.2273 & 0.0595 & 0.0212 & 0.0042 & 0.0369 & 0.3687 & 0.4189 & 0.0090 & 0.6542\end{array}$

Set-2 vs Set-4 $0.3961 \quad 0.0485 \quad 0.0514 \quad \begin{array}{lllllll}0.0008 & 0.0562 & 0.2971 & 0.2035 & 0.1793 & 0.0362\end{array}$

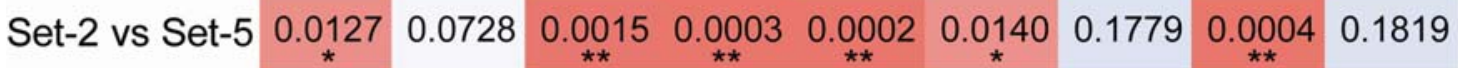

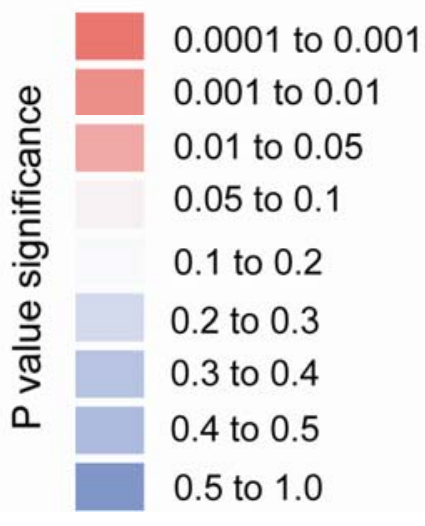


$814 \quad$ Figure $\mathbf{S 3}$

815
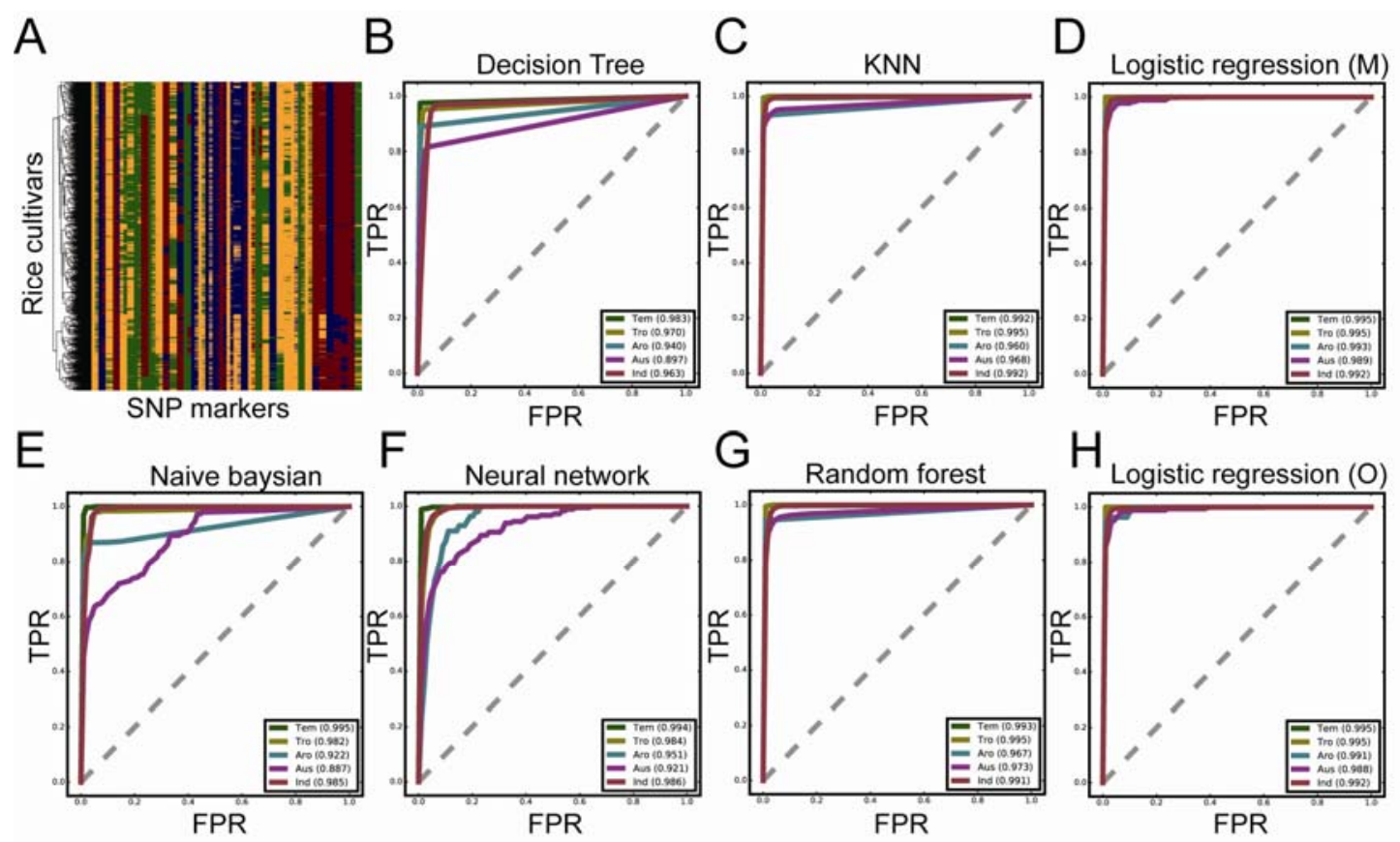

816

817

$818 \quad$ Figure $\mathbf{S 4}$

A

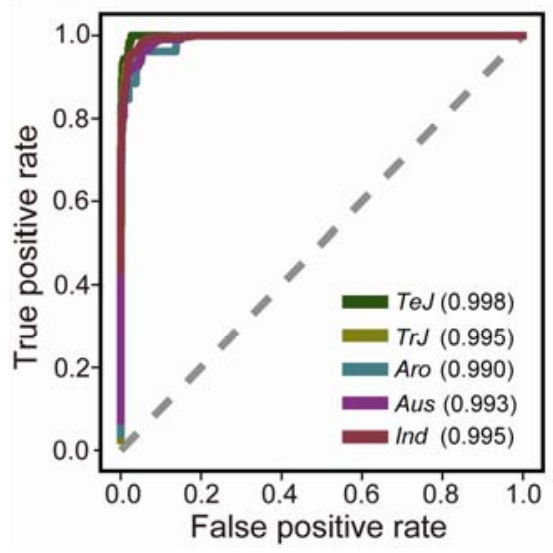

B

\begin{tabular}{lccccc}
\hline & Total & TP & TPR & FP & FPR \\
\hline TeJ & 125 & 121 & $96.80 \%$ & 16 & $2.12 \%$ \\
TrJ & 246 & 228 & $92.68 \%$ & 8 & $1.26 \%$ \\
Aro & 26 & 22 & $84.62 \%$ & 7 & $0.82 \%$ \\
Aus & 112 & 102 & $91.07 \%$ & 14 & $1.82 \%$ \\
Ind & 371 & 351 & $94.61 \%$ & 11 & $2.16 \%$ \\
Total & 880 & 824 & $93.64 \%$ & 56 & $1.59 \%$ \\
\hline
\end{tabular}

